\title{
Dendritic cells in blood and urine samples from bladder cancer patients undergoing BCG immunotherapy
}

\author{
Raffaella Rossi ${ }^{1}$, Miriam Lichtner ${ }^{2}$, Francesco Iori ${ }^{3}$, Angela Ermocida ${ }^{1}$, \\ Claudia Mascia ${ }^{1}$, Fabio Mengoni ${ }^{1}$, Ilaria Sauzullo ${ }^{1}$, Danilo Dini ${ }^{3}$, \\ Claudio M. Mastroianni ${ }^{2}$, Vincenzo Vullo ${ }^{1}$ \\ ${ }^{1}$ Department of Public Health and Infectious Diseases, "Sapienza" University, Rome, Italy; \\ 2 Infectious Diseases Unit "Sapienza" University, Latina, Italy; \\ ${ }^{3}$ Department of Urology, "Sapienza" University, Rome, Italy.
}

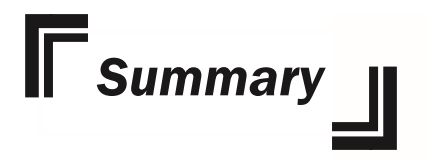

Objectives: Immunotherapy with BCG (Bacille Calmette-Guérin) after transurethral resection of the bladder tumor represents a highly effective primary treatment for intermediate and high-risk superficial bladder cancer. The effectiveness of this therapy has been documented, but its mechanism of action is not clear yet. In the present study, we investigated the changes of dendritic cells (DC) numbers in peripheral blood and urine of patients with superficial bladder cancer undergoing BCG intravescical therapy Material and method: We have enumerated plasmacytoid and myeloid DCs in the peripheral blood and in the urine of patients with bladder cancer in order to clarify the role of these cells in the evolution of the disease and the effect of therapy. DCs in blood and urine samples were assessed using the single-platform TruCOUNT assay with monoclonal antibodies. The study population included 37 healthy donors and 13 patients with diagnosis of primitive superficial bladder cancer.

Results: At the time of diagnosis a reduction of blood DCs was found in patients as opposed to healthy donors, while DCs were not found in the urine in the same way as in healthy subjects. Six of these patients were followed before and after weekly and monthly instillations of BCG. In the peripheral blood, we observed an immunological recovery of DCs from the third weekly instillation up to the sixth. In the urine of patients, we didn't find $\mathrm{mDCs}$ or pDCs at T0, but we found a statistically significant change from the third instillation up to the sixth. On the contrary, we didn't find $m D C$ s in urine during monthly instillation.

Conclusions: DC Count could be used in the monitoring of patients undergoing BCG therapy. Immunological restoration of $\mathrm{mDC}$ numbers in peripheral blood and the efflux in urine could be important for confirming the effectiveness of BCG instillation.

KEY WORDS: Dendritic Cells; TruCount assay; BCG therapy; Superficial Bladder Cancer; Urine.

\section{INTRODUCTION}

The efficacy and the cost-effectiveness analysis of Bacille Calmette-Guérin (BCG) therapy in the management of high-risk superficial bladder cancer (NMIBC) or treatment of carcinoma in situ (Tis) has been demonstrated (1-3). The goal of BCG therapy is to eradicate the disease and to inhibit tumor recurrence and prevent the progression of cancer (4-6). Although used in large number of patients, the immunotherapy mechanism of action of BCG has remained a black box for three decades. A local inflamma- tion, characterized by an influx of mononuclear cells into the bladder wall and by the secretion of pro-inflammatory cytokines into the urine, has been described in patients undergoing BCG instillations (7-9). Recently, a central role in the prevention bladder tumor recurrence has been suggested for granulocyte-macrophage colony-stimulating factor (GM-CSF) and tumor necrosis factor (TNF)- $\alpha$, which are essential cytokines in the induction of dendritic cell (DC) response (10). In fact, both natural and adap- 
tative immunity seem to be involved in the local response to BCG therapy. Several studies showed the presence of natural killer (NK) cells, CD4+ and CD8+ T lymphocytes in the bladder wall in patients with superficial transitional cell carcinoma (TCC) undergoing BCG instillations. This presence was correlated with a reduction in superficial bladder cancer recurrence (11). Few studies have examined the significance of the presence of dendritic cells (DC) in the urine from patients with bladder cancer. The importance of DCs as immunotherapy against cancer has been widely studied (12-14). In fact, DCs are considered professional antigen presenting cells (APCs) for inducing anticancer immunity, both in vitro and in vivo (15), and they can induce both primary and secondary immune responses. Nishiyama et al, propose DC-based cancer immunotherapy as an additional treatment against advanced bladder cancer (16). Cheadle et al. demonstrated in vitro that BCG-infected DC are potent activators of T-cells and adaptive immune response (17). Two types of immature circulating DCs were found in human blood: myeloid DCs (mDCs) and plasmacytoid DCs (pDCs) that can be identified for their phenotypic markers and different function (18). A recent study tested the hypothesis that DCs may also migrate in the urine of bladder cancer patients. The authors speculate that variability in the percentage of urinary DCs may reflect changes in immunological activity at the tumor site (19). However, they did not enumerate circulating DCs in the periphery. In this study, we used a single-platform flow cytometric Tru-
COUNT assay to count the absolute number of the two subsets of DCs in both peripheral blood and urine samples from patients with bladder cancer before transurethral resection. In addition, in a longitudinal study, the enumeration of mDCs and pDCs count was done at different times of weekly and monthly BCG instillation.

\section{Materials AND Methods}

The study population included 13 patients with diagnosis of primitive bladder cancer, confirmed by transurethral resection bladder (TURB) (10 males, 3 females; age range, 55-81 years old). TNM (T: size or direct extent of the primary tumor. $\mathrm{N}(0-3)$ : degree of spread to regional nodes. M (0/1): presence of metastasis) classification of tumors in studied patients was as follows: TaG1 in 1 patient, TaG2 in 3 patients, TaG3 in 5 patients, T1G3 in 3 patients and T2G3 in 1 patient. Six patients ( 3 with TaG2 and 3 with TaG3) were repeatly analyzed during weekly and monthly instillations with BCG, according to the Lamm's protocol (20). All patients were admitted to the Department of Urology of the Azienda Policlinico Umberto I, Sapienza University of Rome. Twentysix healthy donors were included as control group. Informed consent was obtained from all subjects before being included in the present study. The study was approved by the institutional review board (Department of Infectious and Tropical Diseases, Sapienza University of Rome). Blood and urine were collected in the first morn-
Figure 1.

Cytofluorimetric analysis of DC subsets in blood sample using TruCOUNT assay.

This is one representative example of DC Count in a healthy donor and a patient with superficial bladder cancer.

In R1 Gate we have identified lymphocytes and monocytes (PBMCs), using as parameters the Side Scatter Channel (SSC) and the CD45-PerCP.

Beads are on the right of the dot plot (a).

Gate $R 2$ recognized PBMCs that were lineage-negative (b). Gate $R 4$ represents TruCOUNT beads events and it was obtained in an ungated dot plot of FL1 vs. FL2 (c).

Finally, to define $m D C$ and $p D C$, events from $R 1$ and $R 2$ were analyzed in a contour plot of $C D 11 C$ or $C D-123$ vs. HLA-DR (d). All CD11chi-HLA-DR+ and CD123hi-HLA-DR+ were included in this gating strategy.

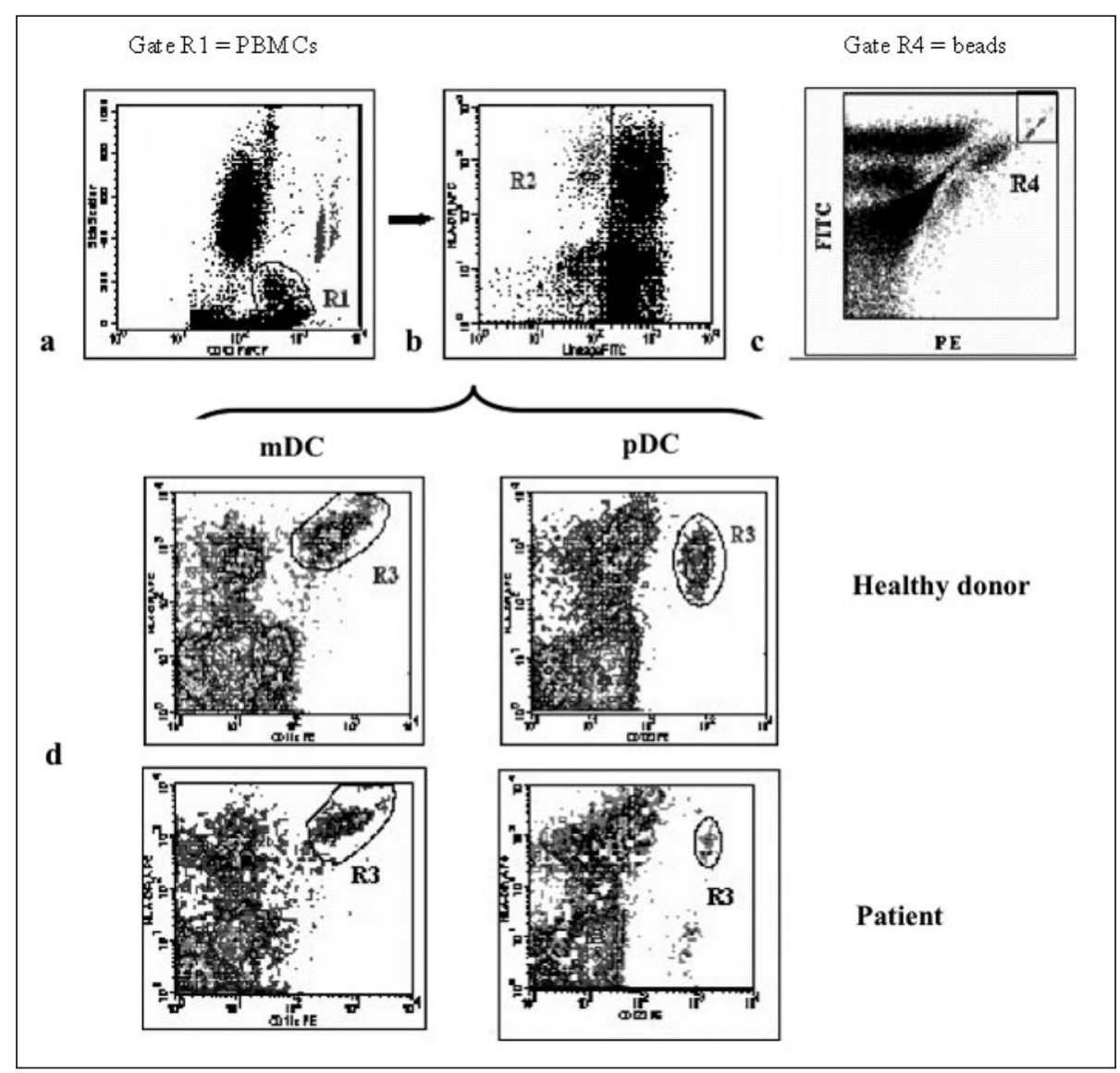


ing before TURB. All patients had no change in lymphocytes and monocytes values. To identify DC subsets, we used a new single platform flow cytometric assay, based on TruCOUNT TM tubes, which contain a known number of fluorescent beads as inside control. This method has many advantages: first of all, we use whole blood and not conventional ficoll-density separation. The absolute number of $\mathrm{pDCs}$ or $\mathrm{mDCs}$ (cells/ml blood) was calculated by the following formula: ( $\mathrm{mDC}$ or $\mathrm{pDC}$ events $\mathrm{x}$ known TruCOUNT beads)/ (beads events x $0.1 \mathrm{ml}$ ). In this way, we eliminate the count obtained from haematology blood analyzer data. Besides, this count is highly reproducible with intra and inter assay, and fast to execute. For DC enumeration, peripheral blood was conserved in EDTA (ethylenediaminetetraacetic acid) tubes. Whole blood $(0.1 \mathrm{ml})$ was directly labeled in TruCOUNT TM tubes, adding monoclonal antibodies (mAbs) and isotype control and using Lyse/No Wash Assay. mAbs for the labelling were: anti CD45-PerCP to identify peripheral blood mononuclear cells (PBMCs), anti HLA-DR-APC that marks DCs and activated cells, Lineage-FITC cocktail (composed of anti-CD3, antiCD14, anti-CD16, anti-CD19, anti-CD20, anti-CD56) because DCs do not express this marker, anti-CD11c-PE or anti-CD123-PE specific markers for mDCs or pDCs respectively. Finally Mouse anti-IgGla-PE and Mouse anti-IgG2a-PE were used for isotype control. Gating strategy is shown in Figure 1. All antibodies and TruCOUNT TM tubes were purchased from Becton
Dickinson (BD Biosciences Pharmingen, Italy). After mixing, the tubes were incubated for 15 minutes in the dark at room temperature (RT); then $450 \mu$ l of FACS Lysing Solution (BD) was added to each tube, after which they were vortexed and incubated for 15 minutes at RT. As for urine, before the staining of DCs, the samples were concentrated 1:100. Then, mAbs and isotype control were added following the protocol for DCs staining in peripheral blood (Figure 2). All samples were analyzed within 1-3 h of staining using a FACScalibur flow cytometer and CellQuest 1.0, and 100.000 events were acquired (Becton Dickinson, Mountain View CA). All data were collected using identical instrument settings. For statistical analysis Sigma Stat 2.2 (Jandel Scientific Software, San Rafael, CA) was used. Values are given as median and ranges. The statistical differences of values were analyzed using the nonparametric Mann-Whitney $U$ test and Spearman coefficients were calculated to measure the association among parameters.

\section{RESULTS}

\section{Cross-sectional study}

The enumeration of both mDCs and pDCs was performed in peripheral blood and urine samples from 13 patients before transurethral resection (Time 0 ). mDCs and pDCs were significantly reduced in peripheral blood from patients with superficial bladder cancer in comparison

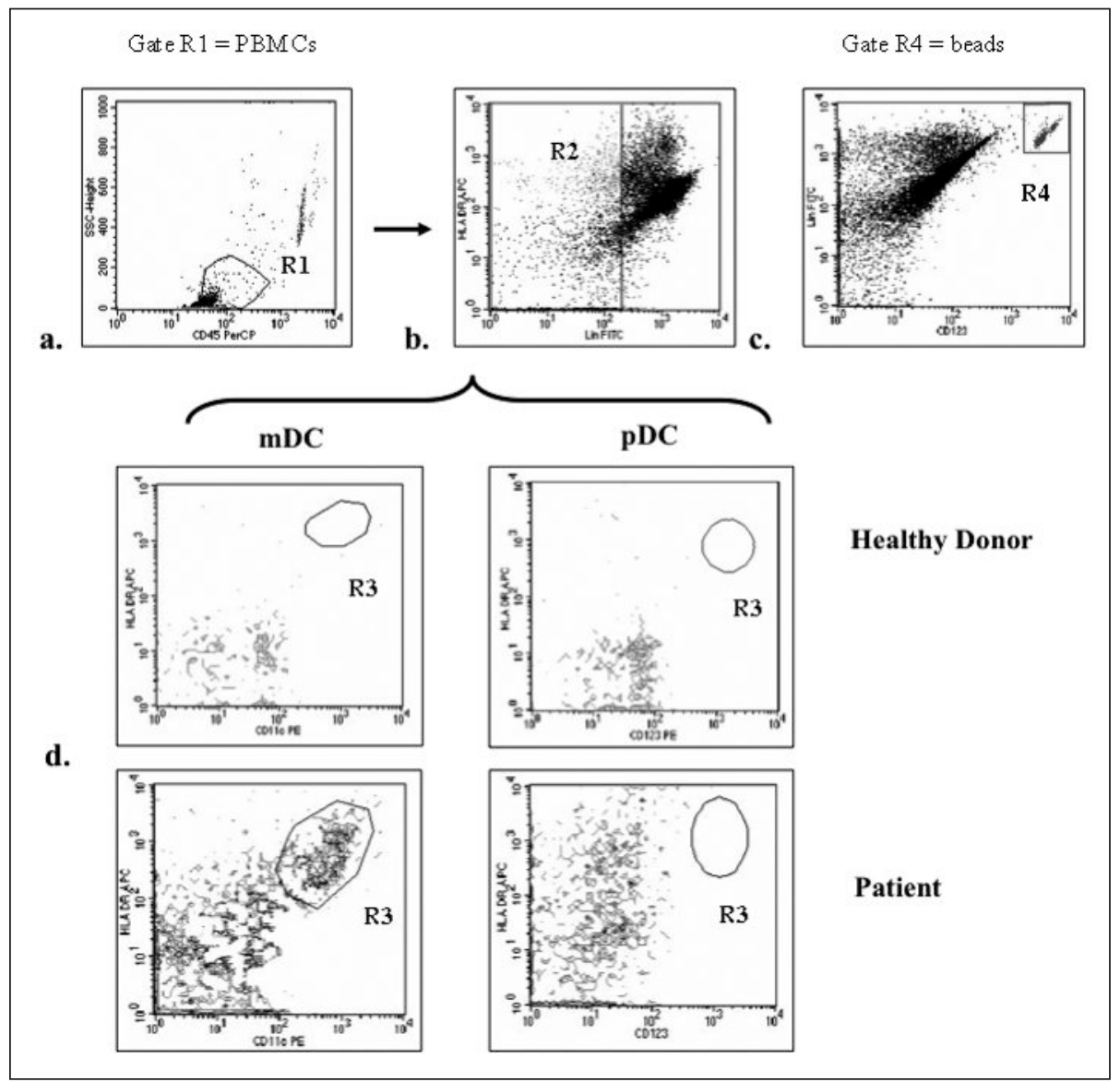

Figure 2.

Cytofluorimetric analysis of $D C$ subsets in urine sample using TruCOUNT assay. This is one representative example of DC Count in healthy donor and patient with superficial bladder cancer. The same strategy of blood sample was used and the figure shows the contour plot of $\mathrm{MDCs}$ and $p D C$ s events (Gate R3) in the urine. 
Figure 3.

Circulating $m D C$ and $p D C$ Count in peripheral blood. Two subsets of DCs were measured using TruCOUNT assay and asignificant reduction in both $\mathrm{mDCs}(\mathrm{a})$ and $\mathrm{pDCs}(\mathrm{b})$ was found in the patients.

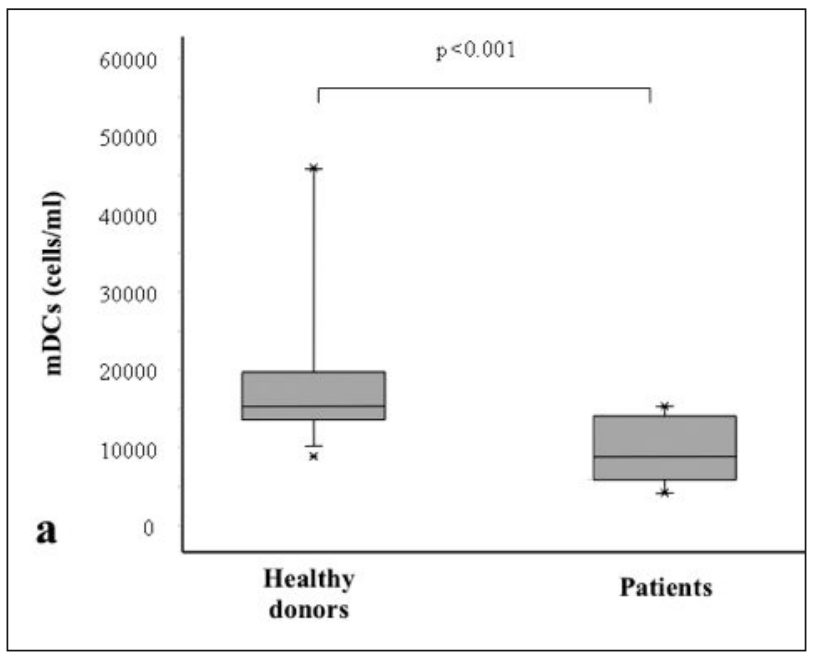

with healthy donors. In fact, the median value of mDCs in patients was 8825 cells/ml (range 4187-15317) vs 15300 cells/ml (8901-45917) in healthy donors ( $p<0.001)$. Similarly, a decrease in pDC count was found (bladder cancer: 5411 cells/ml, range: 2897-10693; healthy donors: 13553, 3875-52111; p < 0.001) (Figure 3).

When patients were stratified on the basis of cancer grading, we observed a significant reduction of mDCs ( $p=0.004)$ in low Grade groups (G1 and G2) when compared with the higher Grade group (G3). No significant differences were found in pDCs count ( $p=0.918$ ) between the two groups. When urine samples were assessed by cytofluorimetric analysis, we did not detect

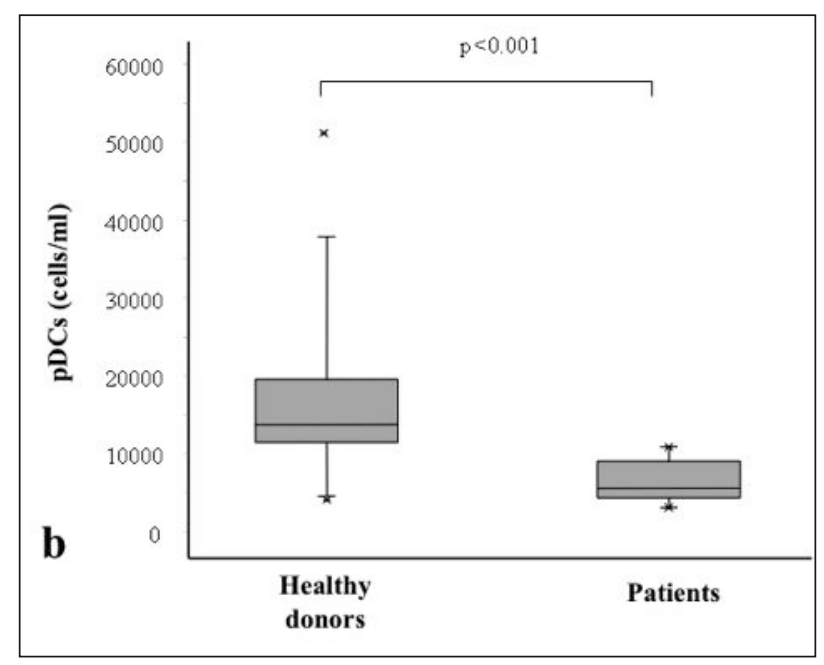

the presence of mDCs and pDCs either in patients or healthy donors.

\section{Longitudinal study}

After transurethral resection, six patients were followed during weekly and monthly instillations with BCG. During a median follow up of 24 months, all patients were free of recurrences. We counted DCs subpopulations in blood and urine before and after 24 hours of the first (T1-T2), third (T3-T4), fifth (T5-T6) and sixth (T7T8) weekly instillations and before and after 24 hours of the sixth monthly instillation (T9-T10). In the peripheral blood, the patients had a recovery of mDCs respect to

Figure 4.

Circulating $m D C$ and $p D C$ Count in peripheral blood post-instillations. mDCs and pDCs were measured using TruCOUNT assay. A significant increase was found in $\mathrm{mDC}$ at $\mathrm{T} 8$ of weekly instillations compared to TO (a). No significant differences in pDC Count were found (b). Lines - represent the median values of mDCs and pDCs in the healthy donors.
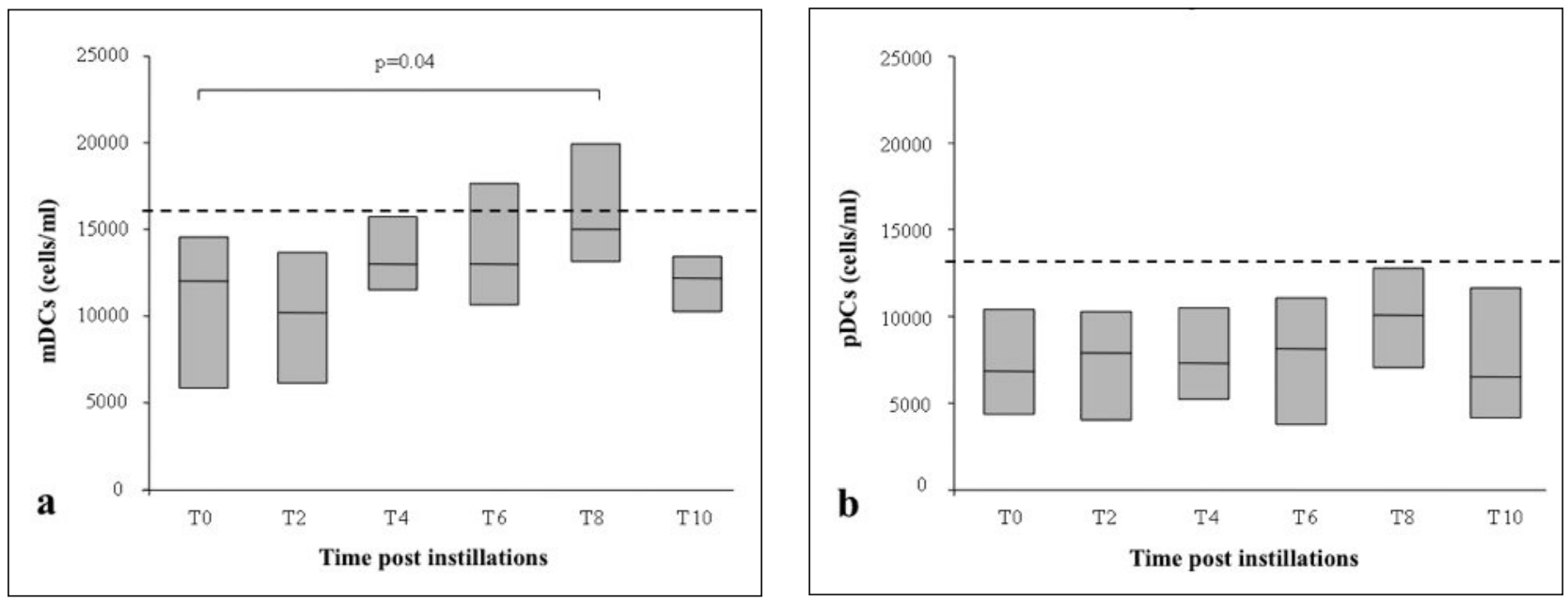
Figure 5.

mDC Count in the urine, post-instillations, using TruCOUNT assay. A significant increase was found in $m D C$ s from the third up to the sixth weekly instillations (T4, T6 and T8) compared to TO.

No statistically increase was found at sixth monthly instillation (T10) if compared to TO. Lines - represent the median values of $m D C s$ in the healthy donors.

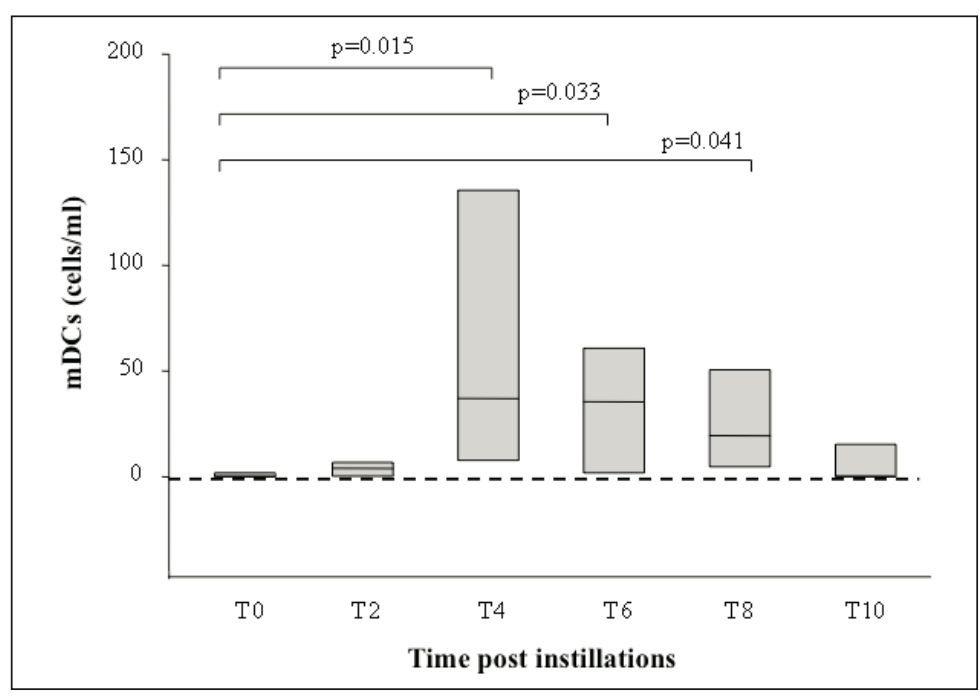

or lamina propria and frequently have a good response to therapy.

The treatment for superficial TCC is transurethral resection bladder (TURB), and $80 \%$ survival is achieved, but less than half are cured because of the relapse (50-70\%). This resection is followed by intravescical therapy that has been shown to be the treatment of choice for intermediate and high superficial bladder cancer. This therapy was first used in the management of bladder cancer in 1976 by Morales et al. and it is an immunotherapy based on instillations with live attenuated tuberculosis vaccine, Bacille Calmette-Guérin (BCG). In the present study, we investigated the changes of DC numbers in peripheral blood and urine of patients with superficial bladder cancer undergoing BCG intravescical therapy (22). We found for the first time a significant decrease of both circulating mDCs and pDCs absolute count in patients with superficial bladder cancer, before transurethral resection, compared to healthy donors. The reduction of DCs in peripheral blood may have functional consequences on DCs activity against the progression of tumor: mDCs play an important role in cancer for their

T0 ( $p=0.04)$ and at the end of weekly instillations, the values were comparable to healthy donors $(p=0.7)$. At the sixth monthly instillation, none of the patients showed numerical reduction of mDCs. There was an increase from the third instillation (Figure 4a). No significant changes were observed before and after 24 hours of the BCG instillations (data not shown). For the pDCs, we found only a partial increase compared to TO at the end of weekly instillations without a statistical significance ( $p=0.2)$ (Figure $4 b)$. At the sixth monthly instillation, the pDC values decreased to the pre-instillation levels, indicating that peripheral blood pDCs were partially affected by BCG instillation. The analysis of the urine showed that $\mathrm{mDC}$ were detectable in the urine from the third instillation up to the sixth instillations. No changes were found before and after 24 hours of the BCG instillations. In fact, during the first instillation we didn't find mDCs before and after 24 hours. Otherwise, at the third instillation, $\mathrm{mDC}$ were detected in urine and remained stable until the end of weekly instillation. Little or no $\mathrm{mDC}$ release was found in urine during monthly instillations (Figure 5). Analysis using the Spearman rank correlation test showed that there was no statistically significant correlation between mDCs in urine and blood $(r=0.240, p=0.3)$.

\section{Discussion}

Transitional cell carcinoma (TCC) is found in more than $90 \%$ of patients with bladder cancer, and this type of cancer is either superficial (70\%) or muscle-invasive. Patients with muscle-invasive or metastatic disease have a poor prognosis and they will die within 2-3 years after diagnosis. Superficial tumors are confined to the mucosa antitumor properties, while pDCs are the natural IFNproducing cells in the immune system. On the other hand, when patients were stratified for tumor grading, data showed that lower cancer grading was associated with the lowest count of mDCs in blood, suggesting that DCs deficit depends on a major recruitment of mDCs at the tumor site, rather than on a peripheral disruption. In a longitudinal study, we have followed six patients after transurethral resection and during weekly and monthly instillations of BCG. We observed a numerical recovery of both circulating DCs in these patients at the end of the weekly treatment. The number of DCs increased from the third instillation, and it may represent a good evidence for the efficacy of BCG immunotherapy in immunological recovery. BCG causes a local inflammation and an influx of mononuclear cells into the bladder wall (8). BCG induces maturation and activation of DCs with interaction with TLRs 2 and 9 and with Dectin$1(17,19,24,25,26,27)$ that are important components of the innate immune response. In our patients, mDCs were found in urine after BCG therapy, particularly at the third instillation that corresponds to blood DCs increase. The mDCs found at the site of immunotherapy in those patients with lower grades may be involved in the protective immune responses elicited by the treatment. Many studies underlined the presence of cells, such as NK cells, CD4+ and CD8+, T lymphocytes in the bladder wall, after BCG instillations in patients with superficial TCC. This presence was correlated with a reduction in superficial bladder cancer recurrence (10) but the results were often conflicting $(28,29,30)$. In other studies, pro-inflammatory cytokines (IL-1, IL-2, IL-6, TNF$\alpha$, IFN- $\gamma$ ) were found in urine after BCG instillations $(7,9,10,31)$ but their role in weekly instillations is not 
clear. Recently, some studies correlated the presence of leukocytes in the urine of patients with bladder cancer undergoing BCG therapy with a reduction of recurrence (10). DCs were found in the urine of bladder cancer patients, and it was speculated that variability in the percentage of urinary DCs may reflect changes in immunological activity at the tumor site (32). It is conceivable that the mDCs we found in the urine of patients under BCG immunotherapy may play a crucial role in the antitumor activity of BCG therapy.

They may interact with the NK cells, T cells and other leukocytes and with various cytokines, through a reciprocal cross-talk that activates anti-tumor responses (33, 34). Indeed a very recent report showed that bladder tumour mature dendritic cells and macrophages are predictors of response to BCG therapy (34). In the last years there has been a lot of interest in the evaluation of peripheral blood DC subsets, as an improvement in the enumeration methods has been obtained (35). Using flow cytometry for DC counts, a reduction of circulating $\mathrm{mDCs}$ and $\mathrm{pDCs}$ was demonstrated for the first time in HIV infection (36-38). Several studies have shown that DCs in blood are decreased by viral, parasitic and bacterial infectious diseases $(39,40)$. In addition, recent studies have shown variations of DC counts in peripheral blood in some tumor such as breast cancer in advanced stage $(41,42)$ and in a urological tumor, prostate adenocarcinoma $(43,44)$. DCs are present in very low percentages in peripheral blood, and for this reason it is difficult to obtain a real and reproducible enumeration of these cells. In our study we have obtained an absolute count of DCs using a single platform Tru COUNT assay and a flow cytometric analysis for rare events that gives a standardization of DCs counting in clinical practice (35). All treated patients showed a release of DCs into the urine, with a burst increase at the third instillations. In agreement with previous data, we suggest that BCGmediated antitumor activity may be a localized phenomenon that induces immune responses against cancer, using $\mathrm{mDC}$ antitumor proprieties. The quantification of DCs using a single platform Tru COUNT assay may be very important to understand the mechanism of $B C G$ action and perhaps to monitor the treatment efficacy. Finally, pDCs seem to have a different role: they decreased in patients with superficial bladder cancer, remained low during BCG treatment and they were never found in urine. From these data we can speculate that deficit of pDCs was a characteristic related to other tumor forms (breast, prostate) (41-44) rather than a consequence of the cancer development.

\section{Conclusion}

The results of our study indicate that the number of DCs in blood and urine shows variations during endovescical instillations with BCG in patients after transurethral resection of superficial bladder cancer. mDCs seem to play a crucial role at the tumor site and they were recruited in urine by BCG instillations from the third instillations.

We suggest that mDCs count in urine samples may be used as a marker to better understand the efficacy of BCG treatment.

\section{REFERENCES}

1. Herr HW, Pinsky CM, Whitmore WF, et al. Experience with intravesical bacillus Calmette-Guerin therapy of superficial bladder tumors. Urology. 1989; 25:119.

2. Iori F, Di Seri M, De Nunzio C, et al. Long-Term maintenance Bacille Calmette-Guèrin therapy in high-grade superficial bladder cancer. Urology. 2002; 59:414.

3. Uchida A, Yonou H, Hayashi E, et al. Intravesical instillation of bacille Calmette-Guerin for superficial bladder cancer: cost-effectiveness analysis. Urology. 2007; 69:275.

4. Baniel J, Grauss D, Engelstein D, Sella A. Intravesical bacillus Calmette-Guèrin treatment for stage T1 grade 3 transitional cell carcinoma of the bladder. Urology. 1998; 52:785.

5. Brake M, Loertzer H, Horsch R, Keller H. Recurrence and progression of stage T1, grade 3 transitional cell carcinoma of the bladder following intravesical immunotherapy with bacillus CalmetteGuèrin. J Urol. 1992; 148:797.

6. Obek C, Shelfo SW, Korman HJ, Soloway MS. Intravesical therapy for transitional cell carcinoma of the bladder: the community practice. Urology. 1999; 53:82

7. Bohle A, Nowc C, Ulmer AJ, et al. Elevations of cytokines interleukin-1, interleukin-2, and tumor necrosis factor in the urine of patients after intravesical bacillus Calmette-Guérin immunotherapy. J Urol. 1990; 144:59.

8. De Boer EC, De Jong WH, Van Der Meijden AP, et al. Presence of activated lymphocytes in the urine of patients after intravesical immunotherapy with bacillus Calmette-Guérin. Cancer Immunol Immunother. 1991; 33:411.

9. De Boer EC, De Jong WH, Steerenberg PA, et al. Induction of urinary interleukin-1 (IL-1), IL-2, IL-6, and tumour necrosis factor during intravesical immunotherapy with bacillus Calmette-Guèrin in superficial bladder bladder cancer. Cancer Immunol Immunother. 1992; 34:306.

10. Shintani Y, Sawada Y, Inagaki T, et al. Intravesical instillation therapy with bacillus Calmette-Guerin for superficial bladder cancer: Study of the mechanism of bacillus Calmette-Guerin immunotherapy. Int J Urol 2007; 14:140.

11. Saint F, Patard JJ, Irani J, et al. Leukocyturia as a predictor of tolerance and efficacy of intravesical BCG maintenance therapy for superficial bladder cancer. Urology. 2001; 57:617.

12. Arroyo JC, Gabilondo F, Llorente L, et al. Immune Response induced in Vitro by CD16- and DC16+ Monocyte Derived Dendritic cells in patients with metastatic renal cell carcinoma treated with dendritic cell vaccines. J Clinic of Immunology. 2004; 24:86.

13. Banchereau J, Palucka K. Dendritic cells as therapeutic vaccines against cancer. Nature Med. 2005; 5:296.

14. Barrou B, Benoit G, Ouldkaci M, et al. Vaccination of prostatectomized prostate cancer patients in biochemical relapse, with autologous dendritic cells pulsed with recombinant human PSA. Cancer Immunol Immunother. 2004; 53:453.

15. Ragde H, Cavanaghi WA, Tjoa B cell based vaccines: progress in immunotherapy studies for prostate cancer. J Urol. 2004; 172:2532.

16. Nishiyama T, Tachibana M, Horiguchi Y, et al Immunotherapy of bladder cancer using autologous dendritic cells pulsed with human lymphocyte antigen-A24-specific MAGE-3 peptide. Clin Cancer Res. 2001; 7:23.

17. Cheadle EJ, Selby PJ, Jackson AM. Mycobacterium bovis bacillus Calmette-Guèrin-infected dendritic cells potently activate autologous $T$ cells via a B7 and interleukin-12-dependent mechanism. Immunology. 2003; 108:79. 
18. MacDonald KP, Munster DJ, Clark GJ, et al. Characterization of human blood dendritic cells subsets. Blood. 2002; 100:4512.

19. Yadav M, Schorey JS. The \{beta\}-glucan receptor Dectin-1 functions together with TLR2 to mediated macrophage activation by mycobacteria. Blood. 2006; 108:3168.

25. Seya T, Akazawa T, Uehori J, et al. Role of toll-like receptors and their adaptors in adjuvant immunotherapy for cancer. Anticancer Res. 2003; 23:4369.

20. Lamm DL, Blumenstein BA, Crissman JD, et al. Maintenance bacillus Calmette-Guérin immunotherapy for recurrent Ta, T1 and carcinoma in situ transitional cell carcinoma of the bladder: a randomized Southwest Oncology Group study. J Urol. 2000; 163:1124.

21. Jakse G, Loindi W, Seeber G. Stage T1 grade 3 transitional cell carcinoma of the bladder: an unfavourable tumor? J Urol. 1987; 137:39.

22. Morales A, Eidinger D, Bruce AW. Intracavity bacillus CalmetteGuerin in the treatment of superficial bladder tumors. J Urol. 1976; 116:180

23. Bafica, A, Scanga CA, Feng CG, et al. TLR9 regulates Th1 responses and cooperates with TLR2 in mediating optimal resistance to Mycobacterium tuberculosis. J Exp Med. 2005; 202:1715.

24. Gagliardi MC, Teloni R, Giannoni F, et al. Mycobacterium bovis bacillus Calmette-Guerin infects DC-SING-dendritic cell and causes inhibition of IL-12 and the enhancement of IL-10 production. J Leukocyte Biology. 2005; 78:1.

26. Stenger S, Modlin RL. Control of Mycobacterium tuberculosis through mammalian Toll-like receptors. Curr Opin Immunol. 2002; $14: 452$.

27. Tsuji S, Matsumoto M, Takeuchi O, et al. Maturation of human dendritic cells by cell wall skeleton of Mycobacterium bovis bacillus Calmette-Guerin: involvement of Toll-like receptors. Infect Immun. 2000; 68:6883.

28. Brandau S, Riemensberger J, Jacobsen M, et al. NK cells are essential for effective BCG immunotherapy. Int J Cancer. 2001; 92:697.

29. Ponticello S, Perna F, Maione S, et al. Analysis of local T lymphocyte subsets upon stimulation with intravesical BCG. A model to study tuberculosis immunity. Respir Med. 1989; 98:509.

30. Ratliff TL, Richey JK, Yuan JJ. T-cell subsets required for intravesical BCG immunotherapy for bladder cancer. J Urol. 1993; 150:1018.

31. Saint F, Kurth N, Maille P, et al. Urinary IL-2 for monitoring intravesical bacillus Calmette-Guèrin response of superficial bladder bladder cancer during induction course and maintenance therapy. Int J Cancer. 2003; 107:434.

32. Beatty JD, Islam S, North ME, et al. Urine dendritic cells: a noninvasive probe for immune activity in bladder cancer? BJU Int. 2004; 94:1377.

33. Abadie V, Badell E, Douillard P, et al. Neutrophils rapidly migrate via lymphatics after Mycobacterium bovis BCG intradermal vaccination and shuttle live bacilli to the draining lymph nodes. 2005; Blood. 106:1843.

34. Ayari C, LaRue H, Hovington H. Bladder Tumor Infiltrating Mature Dendritic Cells and Macrophages as Predictors of Response to Bacillus Calmette-Guérin Immunotherapy. Eur Urol. 2009; 55:1386.

35. Vuckovic S, Gardiner D, Field K, et al. Monitoring dendritic cells in clinical practice using a new whole blood single-platform Tru Count TM assay. JIM. 2004; 284:73.

36. Grassi F, Hosmalin A, McIlroy D, et al. Autran B. Depletion in blood CD11c-positive dendritic cells from HIV-infected patients. AIDS. 1999; 13:759.
37. Pacanowski J, Kahi S, Baillet $M$, et al. Reduced blood CD 123+(Lymphoid) and CD $11 \mathrm{c}+$ (Myeloid) dendritic cell numbers in primary HIV-1 infection. Blood. 2001; 98:3016.

38. Servet C, Zitvogel L, Hosmalin A. Dendritic cells in innate immune responses against HIV: Curr Mol Med. 2002; 2:739.

39. Lichtner M, Maranon C, Vidalain PO, et al. HIV type 1-infected dendritic cells induce apoptosis death in infected and uninfected primary CD4 lymphocytes. AIDS Res Hum Retroviruses. 2004; 20:175.

40. Lichtner M, Rossi R, Mengoni F, et al. Circulating dendritic cells and interferon- $\alpha$ production in patients with tuberculosis: correlation with clinical outcome and treatment response. Clin Expl Imm. 2006; 143:329.

41. Manna PP, Mohanakumar T. Human dendritic cell mediated cytotoxicity against breast carcinoma cells in vitro. J Leukoc Biol. 2002; 72:312.

42. Tschoep K, Manning TC, Harlin H, et al. Disparate functions of immature and mature human myeloid dendritic cells: Implications for dendritic cell-based vaccines. J Leukoc Biol. 2003; 74:69.

43. Heiser A, Coleman D, Dannull J. Autologous dendritic cells transfected with prostate-specific antigen RNA stimulate CTL responses against metastatic prostate tumors. J Clinic Invest. 2002; 109:409.

44. Sciarra A, Lichtner M, Autran GA, et al. Characterization of circulating blood dendritic cell subsets DC123+ (Lymphoid) and DC11C+ (Myeloid) in prostate adenocarcinoma patients. Prostate. 2007; 67:1.

\section{Correspondence}

Raffaella Rossi, MD (Corresponding Author)

raffaella.rossi@hotmail.it

Angela Ermocida, MD

angela.ermocida@tiscali.it

Claudia Mascia, MD

claumascia@tiscali.it

Fabio Mengoni, MD

fabio.mengoni@ uniromal.it

Ilaria Sauzullo, MD

ilariasauzullo@libero.it

Vincenzo Vullo, MD

vincenzo.vullo@uniromal.it

Department of Public Health and Infectious Diseases, "Sapienza"

University, Piazzale Aldo Moro 5 - 00161 Rome, Italy

Miriam Lichtner, MD

miriam.lichtner@uniromal.it

Claudio Mastroianni, MD

claudio.mastroianni@uniromal.it

Infectious Diseases Unit "Sapienza" University, Latina, Italy

Francesco Iori, MD

francescoiori@virgilio.it

Danilo Dini, MD

danilo.dini@libero.it

Department of Urology, "Sapienza” University, Rome, Italy, 